\title{
TRIANGULAR REPRESENTATIONS OF SPLITTING RINGS
}

\author{
BY \\ K. R. GOODEARL
}

\begin{abstract}
The term "splitting ring" refers to a nonsingular ring $R$ such that for any right $R$-module $M$, the singular submodule of $M$ is a direct summand of $M$. If $R$ has zero socle, then $R$ is shown to be isomorphic to a formal triangular matrix ring $\left(\begin{array}{c}A^{0} \\ C\end{array}\right)$, where $A$ is a semiprime ring, $C$ is a left and right artinian ring, and ${ }_{C} B_{A}$ is a bimodule. Also, necessary and sufficient conditions are found for such a formal triangular matrix ring to be a splitting ring.
\end{abstract}

1. Introduction and notation. In [5, Theorem 10], we showed that any right nonsingular ring is an essential product of a ring with essential right socle and a ring with zero right socle. [An essential product of two rings is any subdirect product which contains an essential right ideal of the direct product.] Using this result, [5, Theorem 12] reduces the problem of characterizing splitting rings to characterizing those with either essential socle or zero socle. Since the case of essential socle has been taken care of by [4, Corollary 5.4], only the case of zero socle remains. The purpose of this paper is to study the structure of such a splitting ring with zero socle by representing the ring as a formal triangular matrix ring.

In this paper all rings are associative with identity, and all modules are unital. Unspecified modules are right modules; thus any statement about a bimodule ${ }_{c} B_{A}$ refers to the module $B_{A}$ unless the module ${ }_{C} B$ is specifically mentioned. The reader is assumed to be familiar with the standard notions of singular and nonsingular modules; [3] or [4] may be consulted for details.

We give here for reference our notation, which coincides with that in [4]. For any ring $R$, we let $S(R)$ denote the collection of essential right ideals of $R$. The singular submodule of a module $A$ is denoted $Z(A)$, and for a ring $R$, we use $Z_{r}(R)$ in place of $Z\left(R_{R}\right)$. A submodule $A$ of a module $B$ is said to be $\delta$-closed in $B$ provided $B / A$ is nonsingular, and we let $L^{*}(B)$ denote the collection of $\delta$ closed submodules of $B$. Given any submodule $A$ of $B$, there is a smallest $\delta$ closed submodule $C$ of $B$ which contains $A$, and $C$ is called the $\delta$-closure of $A$ in $B$. We note that the $\delta$-closure of a two-sided ideal in a ring $R$ is again a twosided ideal of $R$. For ease of expression, a two-sided ideal of $R$ which belongs to $L^{*}(R)$ is referred to as a "two-sided ideal in $L^{*}(\mathrm{R})$ ". Finally, we use $S^{\circ}$ (or $S_{R}^{\circ}$ ) to stand for the localization functor associated with a right nonsingular ring $R$ : $S^{\circ}$ is an exact functor from right $R$-modules to right modules over the ring $S^{\circ} R$ (which coincides with the maximal right quotient ring of $R$ ).

Received by the editors August 4, 1972.

AMS (MOS) subject classifications (1970). Primary 16A48.

Key words and phrases. Nonsingular ring, singular submodule, splitting properties, splitting ring.

Copyright $\odot$ 1974, American Mathematical Society 
2. The representation of a splitting ring. In this section, we represent a right nonsingular splitting ring $R$ (with zero socle) as a formal triangular matrix ring $\left(\begin{array}{l}A \\ B\end{array}\right)$, where $A$ is a semiprime ring and $C$ is a left and right artinian ring. In order to accomplish this, some sort of chain condition on $R$ is needed. This is provided by Theorem 1, which shows that the prime radical of $R$ is finite dimensional. We conjecture that in fact $R$ itself must be finite dimensional.

Theorem 1. Let $R$ be a right nonsingular splitting ring such that $\operatorname{soc}\left(R_{R}\right)=0$. If $P$ denotes the prime radical of $R$, then $P_{R}$ is finite dimensional.

Proof. Our general procedure is to show first that all nilpotent ideals of $R$ are finite dimensional. This allows us to prove that $\boldsymbol{P}$ has plenty of finite-dimensional submodules, which we use to show that $P$ itself is finite dimensional. Organizationally, the proof consists of a series of lemmas, some of which are proved in slightly more generality than needed here in order to be used in subsequent theorems. We stipulate that Lemmas $\mathrm{C}$ through $\mathrm{J}$ include the hypothesis that $R$ is a right nonsingular splitting ring with $\operatorname{soc}\left(R_{R}\right)=0$.

Lemma A. Let $Z_{r}(R)=0$, and let $N$ be any nilpotent two-sided ideal of $R$. If $M$ is the $\delta$-closure of $N_{R}$ in $R_{R}$, then $M$ is a two-sided ideal in $L^{*}(R)$ whose left annihilator belongs to $S(R)$.

Proof. Letting $H$ denote the left annihilator of $M$, we infer from the nonsingularity of $R$ that $H$ is also the left annihilator of $N$. To get $H \in \delta(R)$, it suffices to show that any element $x \in R \backslash H$ has a nonzero right multiple in $H$. Observing that $x N \neq 0$, we infer that there must be a positive integer $k$ for which $x N^{k} \neq 0$ and $x N^{k+1}=0$. Choosing some $r \in N^{k}$ such that $x r \neq 0$, we see that $x \boldsymbol{r}$ is a nonzero element of $\boldsymbol{H}$.

Lemma B. Let $A$ be any nonsingular right $R$-module with zero socle. If $B$ is any submodule of $A$, then $B$ is the intersection of those essential submodules of $A$ which contain $B$.

Proof. Given $x \in A \backslash B$, let $K$ be maximal among those submodules of $A$ which contain $B$ but not $x$. We claim that $K$ is essential in $A$.

Letting $f: A \rightarrow A / K$ denote the natural map, we see from the maximality of $K$ that $f x$ is a nonzero element of $A / K$ which is contained in every nonzero submodule of $A / K$. Thus $(f x) R$ is a simple, essential submodule of $A / K$. Inasmuch as $\operatorname{soc}(A)=0,(f x) R$ cannot be projective. Recalling that all simple modules are either singular or projective $[4, \mathrm{pp} .55,56]$, we see that $(f x) R$ must be singular. Since $(A / K) /(f x) R$ is singular also, we infer that $A / K$ is singular. Inasmuch as $A$ is nonsingular, we conclude that $K$ is essential in $A$.

Lemma C. Let $M$ be a two-sided ideal in $L^{*}(R)$ whose left annihilator belongs to $S(R)$. Then $M_{R}$ is a direct summand of $R_{R}$. 
Proof. If $H$ denotes the left annihilator of $M$, then $H$ is a two-sided ideal in $\delta(R)$. Inasmuch as $R / M$ is a nonsingular right $R$-module, [4, Lemma 5.2] says that $(R / M) /(R / M) H$ is a projective right $(R / H)$-module. Then $R /(M+H)$ is projective as an $(R / H)$-module, whence $(M+H) / H$ is a direct summand of $R / H$. Thus there exists an element $m \in M$ such that $m^{2}-m \in H$ and $m R+H=M+H$.

From the equation $m^{2}-m \in H$, we obtain $m^{3}-m \in H$. Since $m \in M$, this yields $m^{4}-m^{2}=0$; hence the element $e=m^{2}$ is an idempotent. Observing that $e R+H=M+H$, we multiply this equation on the right by $M$ to obtain $e M=M^{2}$, whence $M^{2}=e R$. Thus $M^{2}$ is a direct summand of $R_{R}$; hence it suffices to show that $M=M^{2}$.

If $M \neq M^{2}$, then according to Lemma $\mathrm{B}, M_{R}$ must have a proper essential submodule $K$ which contains $M^{2}$. We infer that $M / K=Z(R / K)$, from which it follows that $M / K$ is a direct summand of $R / K$. Thus $R$ must have a right ideal $J$ such that $M+J=R$ and $M \cap J=K$. Observing that $M=M^{2}+J M$, we obtain $M \leq J$, which leads to the contradiction $M=K$.

Lemma D. Let e be an idempotent in the ring $Q=S^{\circ} R$ such that $R \cap e Q$ is a two-sided ideal of $R$.

(a) $R e$ is a unital subring of eQe.

(b) $R e$ is an essential right $(R e)$-submodule of eQe.

(c) $Z_{r}(R e)=0$ and $S^{\circ}(R e)=e Q e$.

Proof. (a) We must show that $R e$ is closed under multiplication, and that $e$ is an identity for $R e$. Given any $x \in R$, we have $x(R \cap e Q) \leq R \cap e Q \leq e Q$. Since $R \cap e Q$ is essential in $e Q$, it follows that $x(e Q) \leq e Q$. Therefore $\operatorname{Re} \subset e Q$, from which the required results are immediate.

(b) If not, then $e Q e$ contains a nonzero element $t$ such that $\operatorname{Re} \cap t \operatorname{Re}=0$.

Since $t Q$ is nonzero, it cannot be a singular right $R$-module. Thinking of $t$ as an endomorphism of $Q_{R}$, it follows that ker $t$ cannot be essential in $Q_{R}$. Inasmuch as $R_{R}$ is essential in $Q_{R}$, we infer that $R \cap t^{-1} R$ is essential in $Q_{R}$, and thus that $\left(R \cap t^{-1} R \cap e Q\right) \otimes(1-e) Q$ is essential in $Q_{R}$. Therefore $\left(R \cap t^{-1} R\right.$ $\cap e Q) \otimes(1-e) Q$ cannot be contained in ker $t$. Noting that $(1-e) Q \leq \operatorname{ker} t$ already, we see that $R \cap t^{-1} R \cap e Q \nsubseteq R \cap$ ker $t$. In view of Lemma B, it follows that $R \cap t^{-1} R$ must have an essential submodule $F$ which contains $R \cap$ ker $t$ but not $R \cap t^{-1} R \cap e Q$.

Set $I=\{(x, t x) \mid x \in F\}$ and $J=\left\{(x, t x) \mid x \in R \cap t^{-1} R\right\}$, both of which are submodules of $R^{2}$. Since $F$ is essential in $R \cap t^{-1} R$, it follows that $I$ is essential in $J$, whence $J / I$ is singular. There exists a map $f: R^{2} \rightarrow Q$ given by $f(x, y)$ $=y-t x$, and we check that ker $f=J$. Thus $R^{2} / J$ is nonsingular; hence $J / I=Z\left(R^{2} / I\right)$. Therefore $R^{2}$ must contain a submodule $K$ for which $J+K$ $=R^{2}$ and $J \cap K=I$.

There exists an element $x \in\left(R \cap t^{-1} R \cap e Q\right) \backslash F$. Set $m_{1}=x$ and $m_{2}=t x$, 
and note that $m_{1}, m_{2} \in R \cap e Q$. Also, let $z_{1}$ and $z_{2}$ denote the elements $(1,0)$ and $(0,1)$ in $R^{2}$.

Since $R^{2}=J+K$, we obtain $z_{i}+\left(u_{i}, t u_{i}\right) \in K$ for some $u_{i} \in R \cap t^{-1} R$. Note that $z_{i} m_{i}+\left(u_{i} m_{i}, t u_{i} m_{i}\right) \in K$ also. Observing that $t u_{i} \in R \cap t R$, we obtain $t u_{i} e \in R e \cap t R e=0$, and thus $t u_{i} m_{i}=t u_{i} e m_{i}=0$. Therefore $u_{i} m_{i}$ $\in R \cap \operatorname{ker} t \leq F$, so that $\left(u_{i} m_{i}, t u_{i} m_{i}\right) \in I \leq K$, from which we get $z_{i} m_{i} \in K$.

Now $(x, t x)=z_{1} m_{1}+z_{2} m_{2} \in K$. Since $x \in R \cap t^{-1} R$, we also have $(x, t x)$ $\in J$, whence $(x, t x) \in I$. Thus $x \in F$, which is a contradiction.

(c) Inasmuch as $Q$ is a regular, right self-injective ring, $e Q$ must be a nonsingular injective right $Q$-module. According to [4, Proposition 1.17], $e Q e$ is thus a regular, right self-injective ring. In view of (a) and (b), [4, Proposition 1.16] now says that $Z_{r}(R e)=0$ and $S^{\circ}(R e)=e Q e$.

Lemma E. Let $e$ be an idempotent in $R$ such that $e R$ is a two-sided ideal. If $R(1-e) \in \delta(R)$, then all nonsingular right $(e R e)$-modules are projective.

Proof. Setting $C=e R e$, we see from Lemma D that $Z_{r}(C)=0$ and $S^{\circ} C=e Q e$, where $Q=S^{\circ} R$. Lemma D also shows that $C=R e$, from which we infer that $r \mapsto r e$ is a unital ring map of $R$ onto $C$. Therefore $C \cong R / R(1-e)$. Since $R(1-e) \in \delta(R)$, it follows as in [4, Theorem 5.3] that $C$ is a right perfect ring. According to [1, Theorem $\mathrm{P}]$, this means that all flat right $C$-modules are projective; hence it suffices to show that all nonsingular right $C$-modules are flat. By [4, Proposition 2.1], this is equivalent to showing that $\left(S^{\circ} C\right)_{C}$ is flat and that $\mathrm{GWD}(C) \leq 1$. We shall prove this by showing that all right $C$-submodules of $S^{\circ} \mathrm{C}$ are flat.

Thus consider any $E \leq(e Q e)_{C}$. Noting that $E R$ is a nonsingular right $R$ module and that $H=R(1-e)$ is a two-sided ideal in $\delta(R)$, we obtain from [4, Lemma 5.2] that $E R / E H$ is a projective right $(R / H)$-module. We have an abelian group epimorphism $f: E R \rightarrow E$ given by $f x=x e$, and it is easily checked that the kernel of $f$ is $E H$. Inasmuch as $f(x r)=(f x)(r e)$ for all $x \in E R$ and $r \in R$, we conclude that $E$ must be a projective right $C$-module. Therefore $E_{C}$ is certainly flat.

Lemma F. If $N$ is any nilpotent two-sided ideal of $R$, then $N_{R}$ is finite dimensional.

Proof. Setting $M$ equal to the $\delta$-closure of $N_{R}$ in $R_{R}$, we see from Lemma $A$ that $M$ is a two-sided ideal in $L^{*}(R)$ whose left annihilator belongs to $\delta(R)$. In view of Lemma $C$, there exists an idempotent $e \in R$ such that $e R=M$. Then Lemmas $\mathrm{D}$ and $\mathrm{E}$ say that $Z_{r}(e R e)=0$ and that all nonsingular right $(e R e)$ modules are projective. According to [4, Theorem 2.11], eRe must be finite dimensional as a right module over itself. Letting $Q=S^{\circ} R$, we have $S^{\circ}(e R e)$ $=e Q e$ by Lemma D; hence [4, Theorem 1.26] says that $e Q e$ is a semisimple ring.

There must exist orthogonal idempotents $e_{1}, \ldots, e_{n} \in e Q e$ such that $e_{1}+\ldots$ $+e_{n}=e$ and each $e_{i} Q e_{i}$ is a division ring. Noting that $Q$ is a semiprime ring (because it is regular), we see from [6, Proposition 2, p. 63] that the $e_{i} Q$ are 
minimal right ideals of $Q$. Therefore $e Q$ is a finitely generated semisimple right $Q$-module. Observing that $S^{\circ}(e R)=e Q$, we infer from [4, Theorem 1.24] that $(e R)_{R}$ is finite dimensional, whence $N_{R}$ is finite dimensional.

Lemma G. Let $\boldsymbol{P}$ denote the prime radical of $R$. Then any nonzero submodule of $P_{R}$ contains a nonzero finite-dimensional submodule.

Proof. Let $T$ denote the union of all nilpotent two-sided ideals of $\mathrm{R}$, and note that $T$ is a two-sided ideal. Any nonzero submodule $A$ of $T_{R}$ must have nonzero intersection with some nilpotent two-sided ideal $N$, and $A \cap N$ is a finite dimensional module by Lemma $F$. Therefore every nonzero submodule of $T_{R}$ has a nonzero finite dimensional submodule. To prove that $P_{R}$ satisfies the same property, it suffices to show that $T_{R}$ is essential in $P_{R}$.

Let $H$ be maximal among those two-sided ideals of $R$ containing $T$ for which $T_{R}$ is essential in $H_{R}$. We claim that $P \leq H$.

Suppose not. Inasmuch as $P$ is contained in every semiprime ideal of $R$ [7, Theorem 4.20], $H$ cannot be a semiprime ideal. Thus there exists a two-sided ideal $K$, properly containing $H$, such that $K^{2} \leq H$. Due to the maximality of $H$, $T_{R}$ is not essential in $K_{R}$, from which we infer that $H_{R}$ is not essential in $K_{R}$. Therefore there exists a nonzero element $x \in K$ which has no nonzero right multiples in $H$. Letting $J$ denote the left annihilator of $K$, we infer from the equation $K^{2} \leq H$ that $x \in J \cap K$. But $J \cap K$ is nilpotent and hence contained in $T$, from which it follows that $x \in H$, which is impossible.

Therefore $P \leq H$; hence $T_{R}$ is essential in $P_{R}$.

Lemma $\mathrm{H}$. The ring $Q=S^{\circ} R$ is a splitting ring.

Proof. We first show that $Q \otimes_{R} Q$ is a nonsingular right $R$-module. According to [2, Theorem 1.6], it suffices to show that for any $a \in Q$, the right ideal $I=\{x \in R \mid a x \in R\}$ has a finitely generated essential submodule. Using [4,Theorem 4.6 and Proposition 4.8], we see that $I$ is AFG, i.e., that $I / \operatorname{soc}(I)$ is finitely generated. Inasmuch as $\operatorname{soc}\left(R_{R}\right)=0$, it follows that $I$ is finitely generated. Therefore $\left(Q \otimes_{R} Q\right)_{R}$ is nonsingular. According to [4, Lemma 1.25], it follows that the natural map $Q \otimes_{R} Q \rightarrow Q$ is an isomorphism.

We must show that any short exact sequence $E: 0 \rightarrow C \rightarrow B \rightarrow A \rightarrow 0$ splits, where $A, B$, and $C$ are right $Q$-modules such that $C$ is singular and $A$ is nonsingular. According to [4, Proposition 1.10], $C_{R}$ is singular and $A_{R}$ is nonsingular, whence $E$ splits as a sequence of $R$-modules. Thus we obtain a split exact sequence $E^{*}: 0 \rightarrow C \otimes_{R} Q \rightarrow B \otimes_{R} Q \rightarrow A \otimes_{R} Q \rightarrow 0$ of right $Q$-modules. Inasmuch as the natural map $Q \otimes_{R} Q \rightarrow Q$ is an isomorphism, we infer that $E^{*}$ is naturally isomorphic to $E$, hence $E$ must split.

Lemma I. If $Q=S^{\circ} R$, then $\operatorname{soc}\left(Q_{Q}\right)$ is finitely generated.

Proof. According to [5, Theorem 10], $Q$ is an essential product of a ring with essential right socle and ring with zero right socle. Inasmuch as $Q$ is its own maximal right quotient ring, it follows from [5, Proposition 2] that $Q$ is actually 
a direct product $H \times K$, where $H_{H}$ has essential socle and $K_{K}$ has zero socle. If $J$ denotes the socle of $Q_{Q}$, then we see that also $J=\operatorname{soc}\left(H_{H}\right)$, and $J_{H}$ is essential in $H_{H}$.

It follows from Lemma $H$ that $H$ is a splitting ring; hence [4, Corollary 5.4] says that $H / J$ is a semiprimary ring. Since $Q$ is a regular ring, we infer that $H / J$ is also a regular ring, whence $H / J$ is actually a semisimple ring.

Now suppose that $J_{Q}$ is not finitely generated. Then we can write $J=\bigoplus_{n=1}^{\infty} J_{n}$, where each $J_{n}$ is an infinite direct sum of simple modules. Observing that $H_{H}$ is injective (since $Q_{Q}$ is injective), we infer that for each positive integer $s$, there must exist an idempotent $e_{s} \in H$ such that $e_{s} H$ is an injective hull for $J_{s}$ and $\left(1-e_{s}\right) H$ is an injective hull for $\bigoplus_{n \neq s} J_{n}$. Note that the idempotents $e_{s}$ are mutually orthogonal. Inasmuch as $e_{s} H$ contains the infinite direct sum $J_{s}$, it follows that $e_{s} H$ cannot be semisimple. Thus $e_{s} H \llbracket J$, i.e., $e_{s} \notin J$. Therefore the images of the elements $e_{s}$ in $H / J$ form an infinite orthogonal sequence of nonzero idempotents, which contradicts the fact that $H / J$ is a semisimple ring.

Lemma J. If $P$ denotes the prime radical of $R$, then $P_{R}$ is finite dimensional.

Proof. Set $Q=S^{\circ} R$ and $J=\operatorname{soc}\left(Q_{Q}\right)$, and let $F$ denote the sum of all the finite-dimensional submodules of $P_{R}$. It follows from Lemma $G$ that $F$ is essential in $P_{R}$, from which we infer that $S^{\circ} F=S^{\circ} P$. If $A$ is any finite-dimensional submodule of $P_{R}$, then [4, Theorem 1.24] says that $S^{\circ} A$ is a finitely generated semisimple right $Q$-module; hence $A \leq S^{\circ} A \leq J$. Thus $F \leq J$, and so $S^{\circ} P$ $=S^{\circ} \mathrm{F} \leq S^{\circ} \mathrm{J}$.

According to Lemma $\mathrm{I}, J_{Q}$ is finitely generated. Inasmuch as $Q$ is a regular ring, it follows that $J$ is a direct summand of $Q$, from which we infer that $S^{\circ} J=J$. Therefore $S^{\circ} P \leq J$, whence $S^{\circ} P$ is a finitely generated semisimple right $Q$-module. By [4, Theorem 1.24], $P_{R}$ must be finite dimensional.

Theorem 2. Let $R$ be a right nonsingular splitting ring with $\operatorname{soc}\left(R_{R}\right)=0$. Then $R$ is isomorphic to a formal triangular matrix ring $\left(\begin{array}{l}A \\ B\end{array}\right)$, where $A$ is a semiprime ring, $C$ is $a$ left and right artinian ring, and ${ }_{C} B$ is faithful.

Proof. We first need a maximal element in the collection of of those two-sided ideals in $L^{*}(R)$ whose left annihilators belong to $\delta(R)$. Let $P$ denote the prime radical of $R$, and set $B=\{A \cap P \mid A \in A\}$. Inasmuch as $P_{R}$ is finite dimensional by Theorem $1,\left[4\right.$, Theorem 1.24] says that $L^{*}(P)$ has ACC. Thus $B$ must have a maximal element, which is of the form $M \cap P$ for some $M \in \mathcal{A}$. We claim that $M$ is maximal in $A$.

Consider any ideal $N \in \mathcal{A}$ which contains $M$. Letting $K$ denote the left annihilator of $N$, we see that $N \cap K$ is nilpotent and hence contained in $P$, whence $N \cap K \leq N \cap P$. The maximality of $M \cap P$ implies that $N \cap P$ $=M \cap P$, from which we obtain $N \cap K \leq M$. Observing that $K \in \delta(R)$, we see that $(N \cap K)_{R}$ is essential in $N_{R}$. Inasmuch as $M \in L^{*}(R)$, it follows that $N \leq M$. Therefore $M$ is maximal in $A$. 
We next claim that $R / M$ is a semiprime ring. If not, then it must have a nonzero nilpotent two-sided ideal $N / M$. Letting $T$ denote the $\delta$-closure of $N_{R}$ in $R_{R}$, it follows just as in Lemma A that the ideal $H=\{r \in R \mid r T \leq M\}$ must belong to $\delta(R)$. Inasmuch as the left annihilator $K$ of $M$ also belongs to $\delta(R)$, we see that $R / K$ and $K / K H$ are both singular right $R$-modules, whence $R / K H$ is singular and $K H \in S(R)$. Noting that $K H T=0$, we infer that the left annihilator of $T$ belongs to $\delta(R)$. But then $T \in \mathcal{A}$, which contradicts the maximality of $M$.

According to Lemma $\mathrm{C}$, there exists an idempotent $e \in R$ such that $e R=M$. Since $e R$ is a two-sided ideal, we obtain $(1-e) R e=0$. Therefore $R$ is isomorphic to the ring $\left(\begin{array}{c}A \\ B C\end{array}\right)$, where $A=(1-e) R(1-e), B=e R(1-e)$, and $C=e R e$. Observing that $R / M \cong A$, we see that $A$ is a semiprime ring.

Since $M \in A$, its left annihilator $R(1-e)$ must belong to $\delta(R)$. According to Lemmas $\mathrm{D}$ and $\mathrm{E}, Z_{r}(C)=0$ and all nonsingular right $C$-modules are projective; hence [4, Theorem 2.12] shows that $C$ is left and right artinian. Any $x \in C$ satisfying $x B=0$ must also satisfy $x R(1-e)=0$, and then $x=0$ [because $R(1-e) \in \delta(R)$ ]. Therefore ${ }_{C} B$ is faithful.

3. Formal triangular matrix rings. The purpose of this section is to derive a few basic properties of a formal triangular matrix $\operatorname{ring}\left({ }_{B}^{A} C\right)$. We are mainly interested in when such a ring can be nonsingular, and in finding the maximal quotient ring of such a ring.

Throughout this section, we assume that $A$ and $C$ are rings, that $c_{A}$ is a bimodule, and that $R$ is the ring $\left(\begin{array}{l}A \\ B\end{array}\right)$. In order to avoid some unnecessary complications, we also make the stipulation that ${ }_{C} B$ is faithful.

Proposition 3. (a) $A$ right ideal $I$ of $R$ belongs to $S(R)$ if and only if it contains a right ideal of the form $\left(\begin{array}{l}J_{0} \\ x_{0}\end{array}\right)$, where $J \in S(A)$ and $K_{A}$ is essential in $B_{A}$.

(b) $R_{R}$ is nonsingular if and only if $A_{A}$ and $B_{A}$ are both nonsingular.

$$
\operatorname{soc}\left(R_{R}\right)=\left(\begin{array}{ll}
\operatorname{soc}\left(A_{A}\right) & 0 \\
\operatorname{soc}\left(B_{A}\right) & 0
\end{array}\right) .
$$

Proof. (a) If $I \in S(R)$, then it is easily seen to contain such a right ideal. Conversely, if $I$ contains a right ideal ( $\left(\begin{array}{ll}x_{k} & 0\end{array}\right)$ of the form described, then we easily infer that $\left(\begin{array}{l}\left.\begin{array}{l}J_{0} \\ K\end{array}\right) \\ 0\end{array}\right)$ is essential in $\left(\begin{array}{l}\begin{array}{l}1 \\ B\end{array} \\ 0\end{array}\right)$. Inasmuch as $C^{B}$ is faithful, it follows that

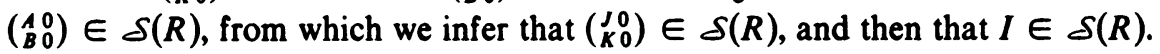

(b) If $R_{R}$ is nonsingular, then it is immediate from (a) that $A_{A}$ and $B_{A}$ are nonsingular. Conversely, assume that $A_{A}$ and $B_{A}$ are nonsingular, and consider

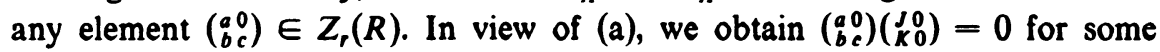
$J \in S(A)$ and some essential submodule $K$ of $B$. We have $a J=0$ and $b J=0$; hence $a=0$ and $b=0$. We also have $c K=0$, whence $c B$ is an epimorphic image of the singular module $B / K$. It follows that $c B=0$, and then the faithfulness of ${ }_{C} B$ implies that $c=0$. Therefore $Z_{r}(R)=0$. 
(c) According to [4, Corollary 1.3], the socle of any module is the intersection of its essential submodules; hence (c) follows immediately from (a).

Let us now assume that $A_{A}$ and $B_{A}$ are nonsingular, so that $R_{R}$ is nonsingular. Set $T=\operatorname{End}_{A}\left(S^{\circ} B\right)$ and $X=\operatorname{Hom}_{A}\left(S^{\circ} B, S^{\circ} A\right)$. Since $c^{B}$ is faithful, we may think of $C$ as a subring of the endomorphism ring of $B_{A}$. Then each element $c \in C$ induces a unique endomorphism $S^{\circ} \mathrm{C}$ of $S^{\circ} B$; hence we obtain an embedding $c \mapsto S^{\circ} c$ of $C \rightarrow T$. For ease of notation, we may thus assume that $C$ is a unital subring of $T$ satisfying $C B \leq B$. We note that the faithfulness of ${ }_{C} B$ is now a consequence of the assumption that $C$ is a subring of $T$.

Proposition 4. $S^{\circ} R=\left(\begin{array}{c}S^{\circ} A \\ S_{B}^{\circ} T\end{array}\right)$.

[Note. To multiply an element $b \in S^{\circ} B$ by an element $f \in X$, we just let $b f$ stand for the map $x \mapsto b(f x)$.]

Proof. Set $Q=\left(S_{S^{\circ} A}^{S^{\circ}} X\right)$. Recalling that the $S^{\circ} A$-homomorphisms from $S^{\circ} A$ to $S^{\circ} A$ or to $S^{\circ} B$ are the same as the $A$-homomorphisms, we see that $S^{\circ} A$ and $S^{\circ} B$ may be identified with $\operatorname{End}_{A}\left(S^{\circ} A\right)$ and $\operatorname{Hom}_{A}\left(S^{\circ} A, S^{\circ} B\right)$. With these identifications, $Q$ is naturally isomorphic to the ring $\operatorname{End}_{A}\left(S^{\circ} A \otimes S^{\circ} B\right)$. Inasmuch as $S^{\circ} A \otimes S^{\circ} B$ is a nonsingular injective right $A$-module, it follows from [4, Proposition 1.17] that $Q$ is regular and right self-injective.

Inasmuch as $A$ is essential in $S^{\circ} A$ and $B$ is essential in $S^{\circ} B$, we see that $R_{R}$ is essential in the module $P_{R}=\left(\begin{array}{c}S^{\circ} A \\ S_{B}^{\circ} C\end{array}\right)$. Now $P$ is also a unital subring of $Q$, and we check that $P_{P}$ is essential in $Q_{P}$, from which it is easy to infer that $R_{R}$ is essential in $Q_{R}$. According to [4, Proposition 1.16], it follows that $S^{\circ} R=Q$.

4. Triangular splitting rings. This section is devoted to developing necessary and sufficient conditions for a formal triangular matrix ring (with zero socle) to be a splitting ring. In light of $\$ 3$, we assume throughout this section that:

(a) $A$ is a right nonsingular ring.

(b) $B$ is a nonsingular right $A$-module.

(c) $C$ is a unital subring of $T=\operatorname{End}_{A}\left(S^{\circ} B\right)$ such that $C B \leq B$.

(d) $R=\left(\begin{array}{ll}A & 0 \\ B & C\end{array}\right)$.

For convenience, we label the following three two-sided ideals of $R$ :

$$
R_{12}=\left(\begin{array}{cc}
A & 0 \\
B & 0
\end{array}\right), \quad R_{2}=\left(\begin{array}{cc}
0 & 0 \\
B & 0
\end{array}\right), \quad \text { and } \quad R_{23}=\left(\begin{array}{ll}
0 & 0 \\
B & C
\end{array}\right) \text {. }
$$

Note that ${ }_{R}\left(R / R_{12}\right)$ and $\left(R / R_{23}\right)_{R}$ are projective, that $R_{12} \in \delta(R)$, and that $R_{23} \in L^{*}(R)$.

Theorem 5. Assume that $\operatorname{soc}\left(R_{R}\right)=0$. If $R$ is a splitting ring, then
(a) $A$ is a splitting ring.
(b) $B_{A}$ is injective.
(c) $C_{C}$ is essential in $T_{C}$.
(d) All nonsingular right $C$-modules are projective. 
(For characterizations of rings satisfying (d), see [4, Theorems 2.11, 2.12, and 2.15].)

Proof. (a) Note that $A \cong R / R_{23}$. Since $R_{23}$ is a two-sided ideal in $L^{*}(R)$, [4, Proposition 1.11] says that the singular submodule of any right $\left(R / R_{23}\right)$-module is the same whether considered as an $\left(R / R_{23}\right)$-module or as an $R$-module. Thus $R / R_{23}$ must be a splitting ring.

(c)(d) The element $e=\left(\begin{array}{l}0 \\ 0 \\ 0\end{array}\right)$ is an idempotent in $R$ such that $e R$ is a two-sided ideal and such that $R(1-e) \in S(R)$. In view of Proposition 4 , we see that $e R e=\left(\begin{array}{ll}0 & 0 \\ 0 & C\end{array}\right)$ and $e\left(S^{\circ} R\right) e=\left(\begin{array}{ll}0 & 0 \\ 0 & T\end{array}\right)$. According to Lemmas $\mathrm{D}$ and $\mathrm{E}$, it follows that $C_{C}$ is essential in $T_{C}$ and that all nonsingular right $C$-modules are projective. For use in the proof of (b), we note that Lemma $D$ also says that $Z_{r}(C)=0$ and that $S^{\circ} \mathrm{C}=T$.

(b) We proceed via several lemmas. With the exception of Lemma $\mathrm{N}$, we stipulate that Lemmas $\mathrm{K}$ through $\mathrm{O}$ all include the hypothesis that $R$ is a splitting ring with $\operatorname{soc}\left(R_{R}\right)=0$.

Lemma K. $S^{\circ} B$ is a finitely generated semisimple right $S^{\circ} A$-module.

Proof. Since $R_{2}$ is nilpotent and therefore contained in the prime radical of $R$, Theorem 1 says that $R_{2}$ is a finite-dimensional right $R$-module. Thus $B_{A}$ must be finite dimensional. According to [4, Theorem 1.24], $S^{\circ} B$ is finitely generated and semisimple.

Lemma L. If $M$ is any simple $S^{\circ} A$-submodule of $S^{\circ} B$, then there exists an idempotent $e \in C$ such that $e\left(S^{\circ} B\right)=M$ and $e T e=e C e$.

Proof. In view of Lemma K, $M$ must be a direct summand of $S^{\circ} B$; hence $M=f\left(S^{\circ} B\right)$ for some idempotent $f \in T$. Inasmuch as the $A$-endomorphisms of $M$ coincide with the $S^{\circ} A$-endomorphisms, we infer that $f T f$ is isomorphic to the ring of $S^{\circ} A$-endomorphisms of $M$, from which it follows that $f T f$ is a division ring. Noting that $T$ is regular and therefore semiprime, we obtain from [6, Proposition 2, p. 63] that $f T$ is a minimal right ideal of $T$.

Observing that $C /(C \cap f T)$ is a nonsingular right $C$-module, we see from (d) that $C \cap f T=e C$ for some idempotent $e \in C$. Since $C_{C}$ is essential in $T_{C}$ by (c), we obtain $C \cap f T \neq 0$, whence the minimality of $f T$ implies that $e T=f T$. Therefore $e\left(S^{\circ} B\right)=f\left(S^{\circ} B\right)=M$. Since $e T$ is a minimal right ideal of $T$, it follows as in the proof of [4, Theorem 2.14] that $e T$ must be a uniserial right $C$ module. In particular, $e C$ must be a characteristic submodule of $e T$, from which we infer that $(e T e)(e C) \leq e C$; hence $e T e=e C e$.

Lemma M. Let $M$ be any simple $S^{\circ} A$-submodule of $S^{\circ} B$. If $I=\{a \in A \mid$ $(M \cap B) a=0\}$, then $M I=0$.

Proof. Set $Q=S^{\circ} R$ and $H=\left(\begin{array}{l}I 0 \\ B 0\end{array}\right)$, and note that $H$ is a two-sided ideal of $R$. Since $S^{\circ} I$ is injective, we must have $S^{\circ} I=f\left(S^{\circ} A\right)$ for some idempotent $f \in S^{\circ} A$. Setting $g=\left(\begin{array}{l}f 0 \\ 01\end{array}\right)$, we infer that $H_{R}$ is essential in $g Q$, from which it 
follows that $S^{\circ} H=g Q$. Thus $R \cap g Q$ is the $\delta$-closure of $H_{R}$ in $R_{R}$, which is a two-sided ideal of $R$.

According to Lemma $\mathrm{D}, R g$ is a unital subring of $g Q g$ and $R g$ is an essential right $(R g)$-submodule of $g Q g$. Observing that

$$
R g=\left(\begin{array}{ll}
A f & 0 \\
B f & C
\end{array}\right) \text { and } g Q g=\left(\begin{array}{cc}
f\left(S^{\circ} A\right) f & f X \\
\left(S^{\circ} B\right) f & T
\end{array}\right),
$$

we infer that $A f$ is a unital subring of $f\left(S^{\circ} A\right) f$ and that $B f$ is an essential right $(A f)$-submodule of $\left(S^{\circ} B\right) f$.

According to Lemma $\mathrm{L}$, there exists an idempotent $e \in C$ such that $e\left(S^{\circ} B\right)$ $=M$. Since $e \in C$, we have $e B \leq B$, whence $e B=M \cap B$. Thus $e B I=0$; hence $e B\left(S^{\circ} I\right)$ is a sum of epimorphic images of the singular module $S^{\circ} I / I$. It follows that $e B\left(S^{\circ} I\right)=0$, whence $e B f=0$. Observing that $e B f$ is an essential right $(A f)$-submodule of $e\left(S^{\circ} B\right) f$, we obtain $e\left(S^{\circ} B\right) f=0$, from which we conclude that $M I=0$.

Lemma N. [For this lemma, we only need the hypotheses that $\operatorname{soc}\left(R_{R}\right)=0$ and that $A$ is a splitting ring.] Let $M$ be any simple $S^{\circ} A$-submodule of $S^{\circ} B$. If $I=\{a \in A \mid M a=0\}$, then $Z_{r}(A / I)=0, M$ is a simple right $S^{\circ}(A / I)$-module, and $S^{\circ}(A / I)$ is a simple artinian ring.

Proof. Inasmuch as $M$ is nonsingular, we see that $I \in L^{*}(A)$. According to [4, Proposition 1.11], it follows that $Z_{r}(A / I)=0$ and that the singular submodule of any right $(A / I)$-module is the same whether considered as an $(A / I)$-module or as an $\boldsymbol{A}$-module. Since $\boldsymbol{A}$. is a splitting ring, it follows that $A / I$ is a splitting ring also. By Proposition 3, we have $\operatorname{soc}\left(A_{A}\right)=0$. Recalling that all simple nonsingular modules are projective $[4, \mathrm{pp} .55,56]$, we infer that the ring $A / I$ must have zero right socle. Setting $P=S^{\circ}(A / I)$, we thus obtain from Lemma $I$ that $\operatorname{soc}\left(P_{P}\right)$ is finitely generated. Inasmuch as $P$ is a regular ring, $\operatorname{soc}\left(P_{P}\right)$ must thus be a direct summand of $\boldsymbol{P}_{\boldsymbol{P}}$.

Noting that $M$ is finitely generated as an $S^{\circ} A$-module, we see from [4, Proposition 1.15] that $M$ is a direct summand of $S^{\circ} B$, from which it follows that $M_{A}$ is injective. Since $M I=0, M$ is also an injective right $(A / I)$-module. Now $M$ is nonsingular as an $A$-module and thus as an $(A / I)$-module; hence we obtain $S_{A / 1}^{\circ} M=M$. Therefore $M$ is a right $P$-module.

Inasmuch as $S_{A}^{\circ} M=M$, the simplicity of $M$ implies that $M$ is indecomposable as an $A$-module, from which we infer that $\mathrm{M}$ must also be indecomposable as a $P$-module. Noting from [4, Proposition 1.15] that all finitely generated $\boldsymbol{P}$ submodules of $M$ are direct summands of $M$, we conclude that $M$ is a simple $P$ module.

Observing that $\{x \in P \mid M x=0\} \cap(A / I)=0$, we obtain $\{x \in P \mid M x$ $=0\}=0$. Therefore $M$ is a faithful simple $P$-module; hence $P$ is a primitive 
ring. In particular, $P$ is a prime ring. The module $M_{P}$ is also nonsingular and simple, hence projective, from which we see that $P$ must contain a minimal right ideal. Thus $\operatorname{soc}\left(P_{P}\right)$ is a nonzero two-sided ideal in the prime ring $P$; hence the left annihilator of $\operatorname{soc}\left(P_{P}\right)$ must be zero. It follows that $\operatorname{soc}\left(P_{P}\right)$ is an essential right ideal of $P$. Since $\operatorname{soc}\left(P_{P}\right)$ is also a direct summand of $P$, we conclude that $\operatorname{soc}\left(P_{P}\right)=P$, i.e., $P$ is a semisimple ring. Inasmuch as $P$ is prime, it must therefore be simple artinian.

Lemma O. $B_{A}$ is injective.

Proof. Suppose not. Since $S^{\circ} B$ is an injective $A$-module, we obtain $B<S^{\circ} B$. In view of Lemma $\mathrm{K}, S^{\circ} B$ must contain a simple $S^{\circ} A$-submodule $M$ such that $M \nsubseteq B$, i.e., $M \cap B<M$. Setting $I=\{a \in A \mid(M \cap B) a=0\}$ and $P$ $=S^{\circ}(A / I)$, we see from Lemmas $\mathrm{M}$ and $\mathrm{N}$ that $M I=0, Z_{r}(A / I)=0, M$ is a simple right $P$-module, and $P$ is a simple artinian ring. Note that since $\{x \in P \mid(M \cap B) x=0\} \cap(A / I)=0$, we obtain $\{x \in P \mid(M \cap B) x=0\}$ $=0$.

According to Lemma $\mathrm{L}$, there exists an idempotent $e \in C$ such that $e\left(S^{\circ} B\right)$ $=M$ and $e T e=e C e$. Inasmuch as $C B \leq B$, we infer that $M \cap B$ is a left $(e T e)$ submodule of $M$.

The $P$-endomorphisms of $M$ coincide with the $(A / I)$-endomorphisms, and the $A$-endomorphisms of $M$ are just the left multiplications by the elements of $e T e$; hence we may identify $e T e$ with the endomorphism ring of $M_{P}$. Since $M_{P}$ is simple and $P$ is a simple artinian ring, we infer that $e T e$ is a division ring, that $M$ is a finite-dimensional left vector space over $e T e$, and that $P$ is the ring of all linear transformations on $M$. However, $M \cap B$ is a proper subspace of $M$, and no nonzero element of $P$ annihilates $M \cap B$, which is impossible.

Theorem 6. Assume that $\operatorname{soc}\left(R_{R}\right)=0$. If the following conditions are satisfied, then $R$ is a splitting ring:

(a) $A$ is a splitting ring.

(b) $B_{A}$ is injective.

(c) $C_{C}$ is essential in $T_{C}$.

(d) All nonsingular right $C$-modules are projective.

Proof. Once again we organize the proof as a series of lemmas. We stipulate that each of Lemmas $P$ through $U$ contains conditions (a)-(d) in its hypotheses.

Lemma P. Any direct sum of copies of $B_{A}$ is injective.

Proof. Since $T$ is the endomorphism ring of the nonsingular injective module $S^{\circ} B,[4$, Proposition 1.17] says that $T$ is a regular, right self-injective ring. In light of condition (c), we see from [4, Proposition 1.16] that $Z_{r}(C)=0$ and $S^{\circ} C=T$. According to [4, Theorem 2.11], condition (d) implies that $C_{C}$ is finite dimensional, whence [4, Theorem 1.26$]$ says that $T$ is a semisimple ring. 
Now there exist orthogonal idempotents $e_{1}, \ldots, e_{n} \in T$ such that $e_{1}+\cdots+e_{n}$ $=1$ and each $e_{i} T e_{i}$ is a division ring. Observing from condition (b) that $S^{\circ} B=B$, we see that $B=e_{1} B \otimes \cdots \otimes e_{n} B$, and that each $e_{i} B$ is an indecomposable $A$-module, hence an indecomposable $S^{\circ} A$-module. According to [4, Proposition 1.15], every finitely generated $S^{\circ} A$-submodule of $B$ is a direct summand of $B$, from which we infer that the modules $e_{i} B$ are simple $S^{\circ} A$ modules.

To show that any direct sum of copies of $B_{A}$ is injective, it suffices to show that any direct sum of copies of $M_{A}$ is injective, where $M$ is any one of the modules $e_{i} B$. Setting $I=\{a \in A \mid M a=0\}$, we obtain from Lemma $N$ that $Z_{r}(A / I)$ $=0$ and that $S^{\circ}(A / I)$ is a simple artinian ring. According to [4, Theorem 1.26], all direct sums of nonsingular injective $(A / I)$-modules are injective. Inasmuch as $M$ is a nonsingular injective $A$-module, it must also be a nonsingular injective $(A / I)$-module; hence any direct sum $\bigoplus M_{i}$ of copies of $M$ must be injective as an $(A / I)$-module. Noting that $\Pi M_{i}$ is an $(A / I)$-module, we infer that $\bigoplus M_{i}$ is a direct summand of $\Pi M_{i}$, from which it follows that $\bigoplus M_{i}$ is injective as an $A$ module.

Lemma Q. If $N$ is any nonsingular right $R$-module, then $N R_{23}$ is a direct summand of $N$.

Proof. The module $N R_{2}$ must be isomorphic to $F / K$ for some direct sum $F$ of copies of $R_{2}$ and some $K \in L^{*}(F)$. In view of Lemma $\mathrm{P}$, we infer that $F$ is injective as a right $\left(R / R_{23}\right)$-module. Inasmuch as $K \in L^{*}(F)$, it follows that $K$ must be a direct summand of $F$, and thus that $N R_{2}$ is injective as an $\left(R / R_{23}\right)$ module. Noting that $N R_{12}$ is an $\left(R / R_{23}\right)$-module which contains $N R_{2}$, we conclude that $N R_{12}=N R_{2} \otimes W$ for some $W$.

Since $R_{2}$ is essential in $R_{23}$, it follows that $R_{23} / R_{2}$ is a singular right $R$-module. Noting that $N R_{23} / N R_{2}$ is a sum of epimorphic images of $R_{23} / R_{2}$, we see that $N R_{23} / N R_{2}$ is singular. Inasmuch as $N R_{23}$ is nonsingular, it follows that $N R_{2}$ is essential in $N R_{23}$. We now take the equation $N R_{2} \cap W=0$ and infer from this that $N R_{23} \cap W=0$. Checking that $N=N R_{23}+N R_{12}=N R_{23}+W$, we conclude that $N=N R_{23} \otimes W$.

Lemma R. If $N$ is any nonsingular right $R$-module, then $N / N R_{12}$ is a projective right $\left(R / R_{12}\right)$-module.

Proof. In view of condition (d), it suffices to show that $N / N R_{12}$ is nonsingular as an $\left(R / R_{12}\right)$-module. Since $N_{R}$ is nonsingular, there exists a monomorphism $N \rightarrow \prod Q_{i}$, where each $Q_{i}$ is a copy of $Q=S^{\circ} R$. Inasmuch as ${ }_{R}\left(R / R_{12}\right)$ is finitely generated and projective, we obtain another monomorphism

$$
N \otimes_{R}\left(R / R_{12}\right) \rightarrow\left(\prod Q_{i}\right) \otimes_{R}\left(R / R_{12}\right) \rightarrow \prod\left[Q \otimes_{R}\left(R / R_{12}\right)\right]
$$


Thus $N / N R_{12}$ is embedded in a direct product of copies of $Q / Q R_{12}$; hence it suffices to show that $Q / Q R_{12}$ is nonsingular as an $\left(R / R_{12}\right)$-module. Using Proposition 4 to check that

$$
Q / Q R_{12}=\left(\begin{array}{ll}
S^{\circ} A & X \\
S^{\circ} B & T
\end{array}\right) /\left(\begin{array}{ll}
S^{\circ} A & 0 \\
S^{\circ} B & 0
\end{array}\right),
$$

we infer that it suffices to prove that $X_{C}$ and $T_{C}$ are nonsingular.

As in Lemma $P$, we have $Z_{r}(C)=0$ and $S^{\circ} C=T$, whence $T_{C}$ is nonsingular. Now consider any eioment $f \in Z\left(X_{C}\right)$. Since $f$ maps $S^{\circ} B$ into the nonsingular module $S^{\circ} A$, we have ker $f \in L^{*}\left(S^{\circ} B\right)$. Inasmuch as $S^{\circ} B$ is injective, it follows that $\operatorname{ker} f=e\left(S^{\circ} B\right)$ for some indempotent $e \in T$. We now infer that $f T$ $\cong(1-e) T$, from which it follows that $(f T)_{C}$ is nonsingular, and thus $f=0$. Therefore $Z\left(X_{C}\right)=0$.

Lemma $S$. Let $n$ be any positive integer, and let $K \in L^{*}\left(B^{n}\right)$. If $J=\left\{x \in C^{n} \mid\right.$ $x B \leq K\}$, then $J B=K$.

Proof. As in Lemma $\mathrm{P}$, we have $Z_{r}(C)=0$ and $S^{\circ} \mathrm{C}=T$. In light of condition (d), we see from [4, Theorem 2.5] that $C$ is right semihereditary and that $Z\left[\left(T \otimes_{C} T\right)_{C}\right]=0$. Then [4, Lemma 2.2] says that ${ }_{c} T$ is flat, while [4, Lemma 1.25] shows that the natural map $T \otimes_{C} T \rightarrow T$ is an isomorphism.

Inasmuch as $B_{A}$ is injective, we have $B=S^{\circ} B$; hence $B$ is a left $T$-module. Then ${ }_{T} B$ is flat because $T$ is a regular ring, and we infer from the flatness of ${ }_{C} T$ that ${ }_{c} B$ must be flat.

Setting $L=\left\{x \in T^{n} \mid x B \leq K\right\}$, we note that $L$ is a right $T$-submodule of $T^{n}$. We have a monomorphism $C^{n} / J \rightarrow T^{n} / L$, from which we obtain another monomorphism $\left(C^{n} / J\right) \otimes_{C} B \rightarrow\left(T^{n} / L\right) \otimes_{C} B$. Now $\left(C^{n} / J\right) \otimes_{C} B$ is naturally isomorphic to $B^{n} / J B$, and we also have natural isomorphisms

$$
\begin{aligned}
\left(T^{n} / L\right) \otimes_{C} B & \rightarrow\left(T^{n} / L\right) \otimes_{T} T \otimes_{C} T \otimes_{T} B \rightarrow\left(T^{n} / L\right) \otimes_{T} T \otimes_{T} B \\
& \rightarrow\left(T^{n} / L\right) \otimes_{T} B \rightarrow B^{n} / L B ;
\end{aligned}
$$

hence we conclude that the natural map $B^{n} / J B \rightarrow B^{n} / L B$ is injective. Therefore $J B=L B$.

Inasmuch as $B^{n}$ is injective and $K \in L^{*}\left(B^{n}\right), K$ must be a direct summand of $B^{n}$. Thus there exists an idempotent $n \times n$ matrix $p$ over $T$ such that $p B^{n}=K$. Given any $x \in K$, we can obtain $x=u_{1} b_{1}+\cdots+u_{n} b_{n}$ for appropriate choices of $u_{i} \in T^{n}$ and $b_{i} \in B$. Since each $p u_{i} B \leq p B^{n}=K$, we see that each $p u_{i} \in L$. Observing that $x=p x$, it follows that $x \in L B$. Therefore $K=L B=J B$.

Lemma T. If $N$ is any nonsingular right $R$-module, then $\operatorname{Tor}_{1}^{R}\left(N, R / R_{23}\right)=0$.

Proof. We may assume, without loss of generality, that $N$ is finitely generated, and we shall prove that the map $N \otimes_{R} R_{23} \rightarrow N$ is injective. Inasmuch as $N R_{23}$ is a direct summand of $N$ by Lemma $Q$, the map $f: N R_{23} \otimes_{R} R_{23} \rightarrow N \otimes_{R} R_{23}$ is 
injective. Noting that $R_{23}$ is idempotent, we see that $f$ is also surjective and hence an isomorphism. Thus it suffices to prove that $N R_{23} \otimes_{R} R_{23} \rightarrow N R_{23}$ is injective.

Since $N$ is finitely generated, we obtain $N R_{23} \cong R_{23}^{n} / H$ for some positive integer $n$ and some $H \in L^{*}\left(R_{23}^{n}\right)$. We check that $H=\left(\begin{array}{l}0 \\ K_{J}\end{array}\right)$ for some $K$ $\in L^{*}\left(B^{n}\right)$ and some $J \leq C^{n}$. Since $H$ is a submodule of $R_{23}^{n}$, we must have $J B \leq K$. Given any $x \in C^{n}$ for which $x B \leq K$, we see that $\left(\begin{array}{l}0 \\ 0 \\ 0\end{array}\right) R_{12} \leq H$. Inasmuch as $R_{12} \in \delta(R)$, it follows that $\left(\begin{array}{l}0 \\ 0 \\ 0\end{array}\right) \in H$, whence $x \in J$. Therefore $J=\left\{x \in C^{n} \mid x B \leq K\right\}$; hence according to Lemma $S$ we obtain $J B=K$.

Now $H R_{23}=\left(\begin{array}{c}0 \\ j B J\end{array}\right)=H$; hence the map $H / H R_{23} \rightarrow R_{23}^{n} /\left(R_{23}^{n}\right) R_{23}$ is injective. Inasmuch as $R_{23}^{n}$ is projective, it follows that $\operatorname{Tor}_{1}^{R}\left(R_{23}^{n} / H, R / R_{23}\right)=0$, i.e., $\operatorname{Tor}_{1}^{R}\left(N R_{23}, R / R_{23}\right)=0$. Therefore $N R_{23} \otimes_{R} R_{23} \rightarrow N R_{23}$ is injective.

Lemma U. $R$ is a splitting ring.

Proof. We must show that $\operatorname{Ext}_{R}^{1}(N, W)=0$ whenever $N$ is a nonsingular right $R$-module and $W$ is a singular right $R$-module. Since it suffices to show that $\operatorname{Ext}_{R}^{1}\left(N, W / W R_{12}\right)=0$ and $\operatorname{Ext}_{R}^{1}\left(N, W R_{12}\right)=0$, we may assume that either $W R_{12}=0$ or $W R_{23}=0$.

Case I. $W R_{12}=0$. Consider any short exact sequence $E: 0 \rightarrow W \rightarrow V \rightarrow N$ $\rightarrow 0$ of right $R$-modules. Since ${ }_{R}\left(R / R_{12}\right)$ is projective, we obtain another exact sequence $E^{*}: 0 \rightarrow W \rightarrow V / V R_{12} \rightarrow N / N R_{12} \rightarrow 0$. According to Lemma $R$, $N / N R_{12}$ is a projective right $\left(R / R_{12}\right)$-module; hence $E^{*}$ splits, from which we infer that $E$ splits.

Case II. $W R_{23}=0$. Consider any short exact sequence $E: 0 \rightarrow W \rightarrow V \rightarrow N$ $\rightarrow 0$ of right $R$-modules. Noting from Lemma $T$ that $\operatorname{Tor}_{1}^{R}\left(N, R / R_{23}\right)=0$, we obtain another exact sequence $E^{*}: 0 \rightarrow W \rightarrow V / V R_{23} \rightarrow N / N R_{23} \rightarrow 0$. Inasmuch as $R_{23}$ is a two-sided ideal in $L^{*}(R)$, [4, Proposition 1.11] says that the singular submodule of any right $\left(R / R_{23}\right)$-module is the same whether considered as an $\left(R / R_{23}\right)$-module or as an $R$-module. In particular, $W$ must be a singular $\left(R / R_{23}\right)$-module. Considering that $N R_{23}$ is a direct summand of $N$ by Lemma $Q$, we see that $N / N R_{23}$ is nonsingular as an $R$-module and hence as an $\left(R / R_{23}\right)$ module. Inasmuch as $R / R_{23}$ is a splitting ring by (a), it follows that $E^{*}$ splits, from which we conclude that $E$ splits.

5. Conclusion. Combining Theorems 2, 5, and 6, we obtain the following structure theorem for splitting rings with zero socle:

Theorem 7. Let $R$ be a right nonsingular ring with zero socle. Then $R$ is a splitting ring if and only if $R$ is isomorphic to a ring of the form $\left(\begin{array}{ll}A & 0 \\ B & C\end{array}\right)$, where

(a) $A$ is a semiprime right nonsingular splitting ring.

(b) $B$ is a nonsingular injective right $A$-module.

(c) $C$ is a unital subring of $T=\operatorname{End}_{A}(B)$.

(d) $C_{C}$ is essential in $T_{C}$.

(e) All nonsingular right $C$-modules are projective. 


\section{REFERENCES}

1. H. Bass, Finitistic dimension and a homological generalization of semiprimary rings, Trans. Amer. Math. Soc. 95 (1960), 466-488. MR 28 \# 1212.

2. V. C. Cateforis, On regular self-injective rings, Pacific J. Math. 30 (1969), 39-45. MR 40 \# 1432.

3. C. Faith, Lectures on injective modules and quotient rings, Lecture Notes in Math., no. 49, Springer-Verlag, Berlin and New York, 1967. MR 37 \#2791.

4. K. R. Goodearl, Singular torsion and the splitting properties, Mem. Amer. Math. Soc. No. 124 (1972).

5. K. R. Goodearl, Essential products of nonsingular rings, Pacific J. Math. (to appear).

6. J. Lambek, Lectures on rings and modules, Blaisdell, Waltham, Mass., 1966. MR 34 \#5857.

7. N. H. McCoy, The theory of rings, Macmillan, New York, 1964. MR 32 \#680.

Department of Mathematics, University of Washington, Seattle, Washington 98195

Current address: Department of Mathematics, University of Utah, Salt Lake City, Utah 84112 